\title{
DEUTSCHES WORTERBUCH von Jacob und Wilhelm Grimm
}

\author{
Petra B. Romanus \\ Oniversidade Federal do Paraná
}

\section{ZUSAMMENFASSUNG}

Es hat uber 100 Jahre gedauert bis das "Deutsche Wörterbuch" von Jacob und Wilhelm Grimm der offentlichkeit ubergeben werden konnte. Am Wörterbuch, konzipiert und angefangen von den Brüdern Grimm, haben Generationen von Philologen gearbeitet, bis es zum grössten und vollständigsten Nachschlagewerk der deutschen Sprache wurde. Es wird heute als das Jahrhundertwerk der deutschen Sprache bezeichnet.

Aus den 6 Bänden und einigen Jahren Arbeitszeit, wie die Brüder das Unternehmen berechnet hatten, wurden iber 30 Bände in einer Zeltspanne von mehr als 100 Jahren. Das Material zum Wörterbuch stammt zum grössten Teil von den Brudern, die schon zu ihren Lebzeiten mehr als 30 Helfer hatten. Andere Verpflichtungen von Jacob und Wilhelm Grimm, politische Unruhen, Kriege und Inflationen haben immer wieder die Fertigstellung des Werkes, 1839 angefangen, verzögert. 1852 konnte endlich die 1 . Lieferung zum Wörterbuch erscheinen. Die Brlider selbst konnten nur die Buchstaben A B C D und F bis zum Wort Furcht fertigstellen; dann nahm ihnen der Tod die Feder aus der Hand.

Das vollständige Werk, eine gewaltige Leistung von Wissenschaftlern aus dem Westen und Osten Deutschlands, besitzt in 38 Bänden die Bundesuniversitat von Paranḱ, vielleicht als einzige in Lateinamerika.

Am Anfang des Jahres 1952 wurde Professor Dr. Reinaldo Bossmann ${ }^{1}$ als Ordinarius auf den Lehrstuhl für deutsche Sprache und Literatur der Bundesuniversität von Paraná be-

1 Ende 1980 emeritiert. 
rufen. Bald danach bat er die Deutsche Forschungsgemeinschaft in Bad Godesberg, dem Lehrstuhl das Wörterbuch der Brüder Grimm als Spende zu beschaffen. Die Deutsche Forschungsgemeischaft entsprach seiner Bitte und schickte in Emzellieferungen, antiquarisch in der Bundesrepublik Deutschland besorgt, so dass später in 38 Baenden das ganze Deutsche Wörterbuch von Jacob (1785-1863) und Wilhelm Grimm (1786-1859) den Dozenten und Studenten zur Verfügung stand. Diese Kostbarkeit - wahrscheinlich die einzige in Südamerika - befindet sich heute in der Zentralbibliothek der Bundesuniversität von Paraná, früher Bibliothek der Philosophischen Fakultät, einschliesslich der bis in die 2. Hälfte dieses Jahrhunderts neugedruckten Lieferungen über die Abhandlung der noch fehlenden Buchstaben und Wörter. Das Werk, von den Brüdern Grimm begonnen, haben Generationen von Philologen bis zur Vollendung fortgesetzt.

Die Erstausgabé des Deutschen Wörterbuchs ist schon lange vergriffen und nur noch gelegentlich antiquarisch zu erwerben. Deshalb bietet jetzt der dtv-Verlag (Deutscher Taschenbuch Verlag) in München einen photomechanischen Reprint der Erstausgabe des Verlages S. Hirzel, Leipzig, als aufgebundene Bibliotheksausgabe und Einzellieferungen an. Dieser Reprint, mit 32 Bänden (rund 70000 Spalten) und 1 Band als Quellenverzeichnis, ist das umfangreichste und bedeutendste deutsche Wörterbuch ïberhaupt. Die nur geringfügig verkleinerte dtv-Ausgabe geht auf die Erstausgabe als Reproduktionsvorlage zuriick und wird viele Bibliotheken der ganzen Welt, die die Erstausgabe besitzen, in Zukunft der Sorge des Zerfalls von Papier und Klebebindung des Originals entheben.

Nach der Entlassung" der Bruider Grimm als Professoren der Universität Göttingen - sie protestierten gegen den Verfassungsbruch des Königs Ernst Augusts von Hannover -, gingen sie ins Exil nach Kassel, um dort ihr weiteres Schicksal abzuwarten. Da sie aber ohne feste Tätigkeit und ohne Einkünfte in Kassel lebten, war ihnen das Angebot der Weidmannschen Buchhandlung in Leipzig im Frïhjahr 1838 willkommen, ein grosses deutsches Wörterbuch zu schaffen. Die Brüder dachten, durch dieses Angebot unabhänging von jeder Staatsstellung leben und ihren wissenschaftlichen Neigungen nach arbeiten zu können. Nach sich in die Länge ziehenden Verhandlungen mit dem Verleger kam es zur Vertragsunterzeichnung. Die Brüder berechneten das Unternehmen auf 6 bis 7 Bände und nahmen eine

2 Ble gehoerten zu den "Goettinger Bleben" (Prolessoren), die thres Amtes im Jahre 1837 enthoben wurden. 
Arbeitszeit von einigen Jahren an. Jacob rechnete mit 6 Jahren, Wilhelm - etwas vorsichtiger - mit 15 Jahren. Aber schon ein Jahr später kamen sie zu der tberzeugung, dass sie sich grundsätzlich in Zeit und Arbeitsmenge verrechnet hatten, ja ob ihr Leben überhaupt dazu ausreichen würde. Als sie die Arbeit begannen, war ja Jacob schon 53 und Wilhelm 52 Jahre alt. Aus den 6 Bänden und den einigen Jahren Arbeitszeit sind über 30 Bände geworden, ein Mammutunternehmen, an dem Generationen von Germanisten mehr als 100 Jahre gearbeitet haben und das mit Recht als das Jahrhundertwerk der deutschen Sprache bezeichnet wird. Das Material zum Wörterbuch ist zum grössten Teil von den Brüdern Grimm selbst gesammelt worden, allerdings stand ihnen eine Reihe von Mitarbeitern zur Verfügung, 1839 sind es schon 30 Helfer gewesen.

Bis zur ersten Lieferung im Jahre 1852 verflossen die Jahre, und ein Bestand an Wörtern, der kaum zu bewältigen war, häufte sich an. Jacob leitete das Unternehmen, war aber durch andere wissenschaftliche Arbeiten beschäftigt. Hinzu kam noch die tbersiedlung der Brider nach Berlin durch die ehrenvolle Berufung an die Universität, die ihnen neue Pflichten und Verpflichtungen brachte: das Einleben in Berlin, das Ausarbeiten von Vorlesungen, die Teilnahme am Gesellschaftsleben in der Preussischen Hauptstadt, politische Ablenkungen wie die Revolution von 1848 und die Berufung von Jacob Grimm als Mitglied der Nationalversammlung zu Frankfurt.

Die Brüder litten unter der Last der Arbeit, die mit dem Wörterbuch auf sie zukam. Sie zu bewältigen, war für die kurze Dauer eines Menschenlebens zuviel. Das geht aus zahlreichen Briefen hervor, die sie an Freunde und Mitarbeiter des Unternehmens geschrieben hatten: "Das übernommene Wörterbuch drückt mit bleierndem Gewicht und ich werde ihm zu Gefallen manches andere mir liebere fahren lassen. Wenn ich bei irgendeiner Arbeit die Schwierigkeit des Anfangs je fühle, so ists bei dieser..." Und an denselben Adressaten am 9.4.1851: "Sie sehn ganz recht, dass mich das Wörterbuch drückt, aber es gibt keinen Ausweg und ich muss eine Art von moralischer Verbindlichkeit erfüllen!"3 Oder: "Das Wörterbuch greift fast zu sehr an, Sie haben ja selbst diese Kost geschmeckt, man ist dabei genötigt auf alles und jedes einzugehn und darf sich nicht bloss auswählen, wofür man sich Neigung und Kenntnisse zutraut..." Am 20.10. 1852 schreibt Jacob Grimm an den Germanisten August Stö-

3 Brief Jacob Grimms an Gerrinius rom 31-1-1850, zitiert nach: GERSTNER. H. Die Brider Grimm; Ihr Leben und Werk. Ebenhausen bel Munchen, Verlag LangewlescheBrandt. 1952. B.276.

4 Jacob Grimm an Weigand, Berlin v.10.1.1852, aus Gerstwhe, B.276. 
ber: "Die allzu lang verschobene Ausarbeitung des deutschen Wörterbuchs hat seit einem Jahr begonnen und ich stecke bis an den Hals in diesem Meer von Arbeit, dass alle meine Kräfte so in Anspruch nimmt, wie ich selbst vorher keine Vorstellung davon hatte. Jeden Tag sitze ich zwölf Stunden dahinter und das will etwas sagen, wenn einer auf dem Sprung steht, achtundsechzig Jahre alt zu werden... Erreicht das Werk, wie es begonnen hat, auch Vollendung, so wird kaum eine moderne Sprache ein so lebendiges von Wörtern und Beispielen aufzuweisen haben. Für den ganzen ersten Band in acht Lieferungen stehe ich mutterallein und kein Buchstab wird darin sein, der nicht aus meiner Feder geflossen wäre"."

Uber das langsame Anwachsen des Wörterbuchs schreibt Jacob Grimm am 14.4.1858 an Dahlmann, indem er folgendes begründet: "Stellen wir uns das Bild des Wörterbuchs einmal lebhaft vor. Ich habe in Zeit von drei Jahren für die Buchstaben A B C geliefert 2464 enggedruckte Spalten, welche in meinem Manuskript 4516 Quartseiten ausmachten. Hier will alles, jeder Buchstabe eigenhändig geschrieben sein, und fremde Hilfe ist unzulässig. Wilhelm wird in drei darauf gefolgten Jahren das $\mathrm{D}$, obschon er es dem Plan entgegen zu sehr ausführt, in 750 Spalten darstellen. Die Buchstaben A B C D erreichen noch nicht ein Viertel des Ganzen. Es bleiben also, mild angeschlagen, noch gegen 13000 gedruckte Spalten oder nach Weise meines Manuspripts 25000 Seiten $\mathrm{zu}$ schreiben. Fürwahr eine abschreckende Aussicht".

An seinem 67. Geburtstage, am 4. Januar 1852, erhielt Jacob den ersten Korrekturbogen vom Verlag zugeschickt. Die erste Lieferung des Wörterbuches erschien im Mai 1852, vierzehn Jahre nach der Unterzeichnung des Vertrages mit dem Verlag. Der erste Band, abgeschlossen gegen Ende 1853, erschien 1854 mit einer langen Vorrede, unterzeichnet von Jacob Grimm am 2. März 1854. Darin schreibt Jacob: "Mag das Werk, dessen Beginn auf des geliebten Vaterlandes Altar wir nun darbringen, einst vollfuihrt gegrïndetere Zuversicht erwecken, dass es im Andenken der Nachwelt haften und nicht schwinden werde, so ist damit alles Leid vergolten... Wie wenn tagelang feine dichte Flocken vom Himmel nieder. fallen, bald die ganze Gegend in unermesslichem Schnee zugedeckt liegt, werde ich von der Masse aus allen Ecken und Ritzen auf mich andringender Wörter gleichsam eingeschneit". Der zweite Band, mit insgesamt 600 Spalten, die Buchstaben $B$ und $C$ behandelnd, wurde bald danach publiziert. Als Wilhelm Grimm seine Arbeit mit dem Buchstaben

6 GERBTNER, \$.279-80. 
D abgeschlossen hatte, starb er am 16. Dezember 1859. Nach dem Tode des Bruder arbeitete Jacob am Buchstaben F. Er kam bis zum Worte FURCHT, als der Tod ihn am 20. September 1863 ereilte und somit ein ubberaus arbeitsreiches Forscherleben beendete.

Die Arbeit em Wörterbuch wurde nun von jüngeren Kräften fortgeführt. Es gab Unterbrechungen und Verzögerungen, bis 1908 die Preussische Akademie der Wissenschaften die Betreuung des Werkes übernahm. Durch 2 Weltkriege und Inflationen traten weitere Verzögerungen auf bis das gewaltige Werk dann nach mehr als 100 Jahren beendet war.

Das Wörterbuch, nach Jacob Grimm, "soll ein Heiligtum der Sprache gründen, ihren ganzen Schatz bewahren, allen zu ihm den Eingang offen halten", beinhaltet den gesamten deutschen Wortschatz von der 2. Hälfte des 15 Jahrhunderts (von Martin Luther) bis zur Gegenwart, sammelt also den ganzen neuhochdeutschen Wortschatz und erläutert ihn etymologisch-geschichtlich. Grundlage, Programm und Muster des Werkes stammen von den Brüdern Grimm. Die Kommentare zu den einzelnen Wörtern haben ein einheitliches Schema: Nach der Verzeichnung des Wortes folgt die Bedeutung durch die lateinische Entsprechung, darauf die etymologische Ableitung, dann die Vorgeschichte des Wortes in der altochdeutschen und mittelhochdeutschen Epoche und schliesslich seine Entwicklung in der neuhochdeutschen Zeit. Für Form und Bedeutung des Wortes werden zahlreiche Belege gebracht.

Die Brüder wollten ein wissenschaftliches Werk, auch zum Nutzen des einfachen Mannes, schaffen. Wilhelm Grimm hat aber mit Recht vorausgesehen, dass es nie die Beliebtheit beim Volke wie etwa die "Kinder - und Hausmärchen" erlangen werde.

\section{RESUMO}

O artigo contém o plano, a história, as dificuldades e interrupçōes na execuçāo do "Dicionário Alemāo" de Jacob e Wilhelm Grimm. Como provam numerosas cartas a cola. boradores e amigos, escritas por Jacob Grimm, este sentiu sensivelmente a pesada carga do trabalho com o dicioná. rio. Jacob previa um tempo de alguns anos, calculando a obra em 6 ou 7 volumes, enquanto seu irmão Wilhelm es. timava o trabalho por 15 anos. Mas os irmãos se engana. ram profundamente com o imenso número de palavras a serem colecionadas, comentadas, historiadas e documentadas. Finalmente reconheceram que uma vida humana inteira ficaria curta demais para esse enorme empreendimento. 
Tendo Jacob iniciado o trabalho em 1839, conseguiu terminar até a sua morte em 1863, as letras A, B, C, deixando incompleta a letra $F$. Seu irmão Wilhelm, que mor. reu em 1859, concluiu a letra $D$.

Geraçōes de filólogos, num trabalho secular, depois de muitas demoras e interrupçōes, causadas pelas duas guerras mundiais e pelas duas inflaçōes na Alemanha, concluiram, na segunda metade do nosso século, o maior e mais importante dicionário da língua alemã, com mais de 30 volumes.

"O dicionário alemão" de Jacob e Wilhelm Grimm, doado à Universidade Federal do Paraná pela Sociedade Alemā de Pesquisa, em 38 volumes - talvez o único na América do Sul - acha-se na Biblioteca Setorial no Edi. fício D. Pedro I.

\section{BIBLIOGRAPHIE}

1 GERSTNER, H. Die Brüder Grimm; ihr Leben und Werk. Ebenhausen bei München, Verlag Langewiesche-Brandt, 1952.

2 GRIMM, J. GRIMM, W. Deutsches Wörterbuch. Lelpzlg, Verlag S. Hirzel, 19594, 38 v.

3 KLUGE, M. RADLER, R. Hauptwerke der deutschen Literatur. München, verlegt bei Kindler, 1974.

4 WEINREICH, H. Die Wahrheit der Wörterbulcher. Die Zeit, 4 Juli 1975. N. 27. 